Supporting Information for

Fluence-Dependent Morphological Transitions in Laser-Induced Graphene Electrodes on Polyimide Substrates for Flexible Devices

Moataz Abdulhafez ${ }^{1}$, Golnaz N. Tomaraei ${ }^{1}$ and Mostafa Bedewy ${ }^{1,2,3, *}$

${ }^{1}$ Department of Industrial Engineering, University of Pittsburgh, 3700 O'Hara Street, Pittsburgh, PA 15261, USA

${ }^{2}$ Department of Chemical and Petroleum Engineering, University of Pittsburgh, 3700 O'Hara Street, Pittsburgh, PA 15261, USA

${ }^{3}$ Department of Mechanical Engineering and Materials Science, University of Pittsburgh, 3700 O’Hara Street, Pittsburgh, PA 15261, USA

*author to whom correspondence should be addressed.

E-mail address: mbedewy@pitt.edu (M. Bedewy) 

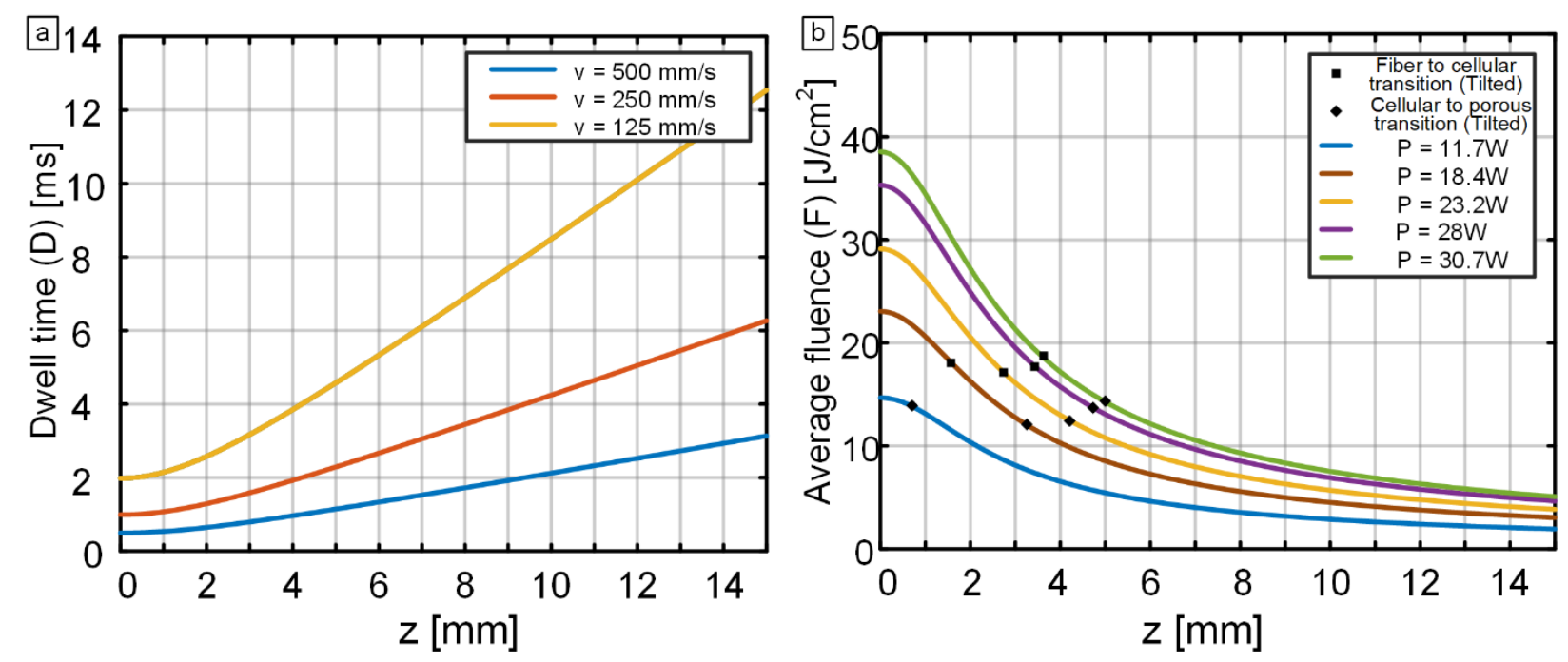

Figure S1. (a) Plot illustrating the change in dwell time with speed and spot size. (b) Plot of estimated average fluence with changing spot size and power for lasing tilted at angle $\gamma=45^{\circ}$. The $\mathrm{x}$-axis represents the equivalent $\mathrm{z}$ values to $\mathrm{x}$ '. 


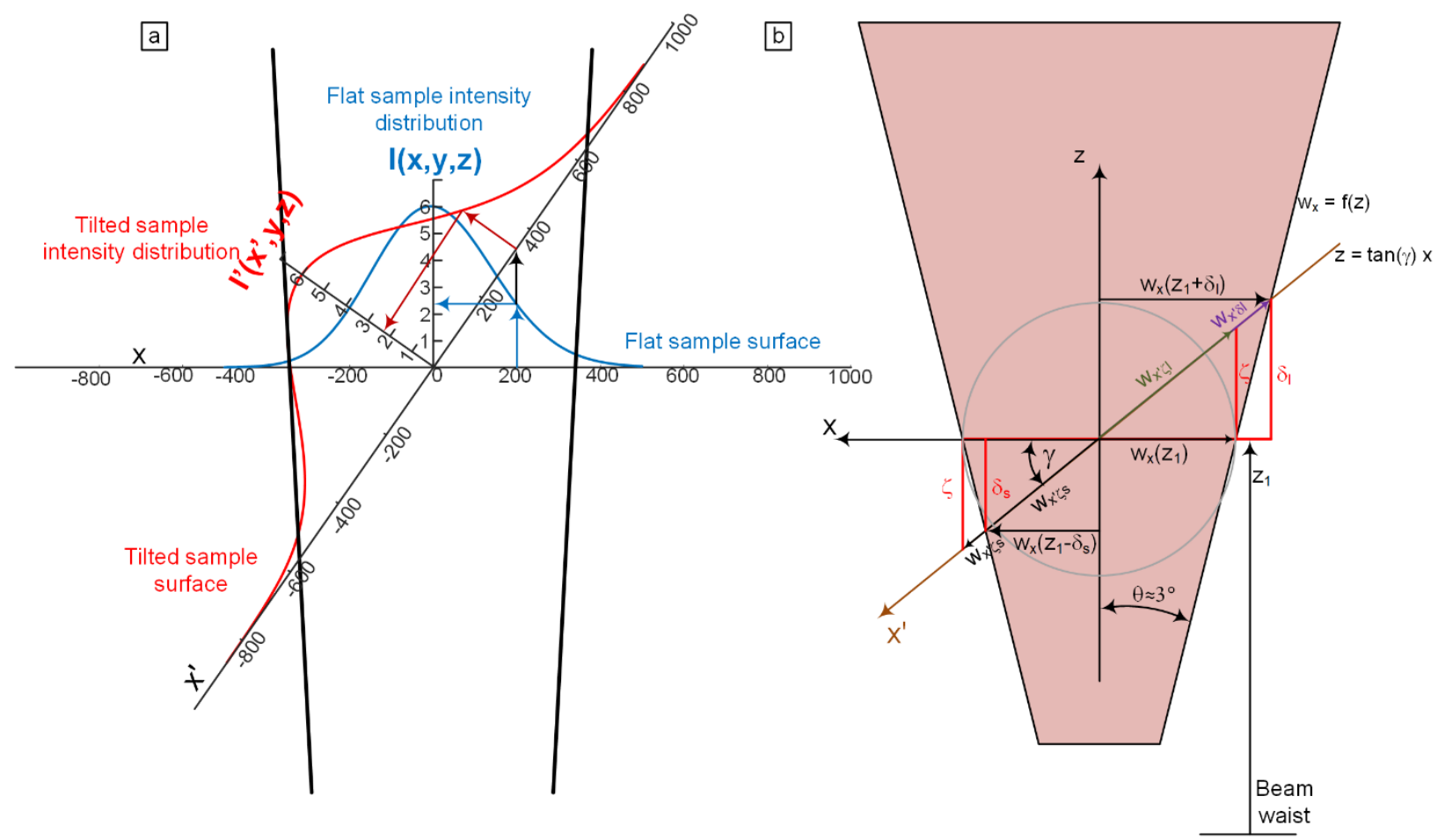

Figure S2. (a) Schematic illustrating the distribution of intensity of laser on a flat untilted sample vs on a tilted sample. (b) Schematic illustrating a section of the beam to show the change of lased area geometry between horizontal and samples tilted with angle $\gamma$.

\section{The effect of tilting on average fluence}

We want to calculate the average energy delivered at each point. This can be calculated by multiplying the average intensity I across the spot size with the dwell time at that point. Since the surface is tilted with angle $\gamma$ : 
- The distribution of flux is not Gaussian (I'( $\left.\left.\mathrm{x}^{\prime}, \mathrm{y}, \mathrm{z}\right)\right)$ across the surface as shown in SI Figure S2(a)

- The dwell time $\mathrm{D}_{t}$ is not constant at the different points along the $\mathrm{x}^{\prime}$ direction

We start be developing a coordinate transformation between the vertical dimension $\mathrm{z}$ and the direction along the tilted sample $x^{\prime}$ :

$$
\begin{gathered}
\tan (\gamma)=\frac{z}{x} \\
\cos (\gamma)=\frac{x}{x^{\prime}} \\
\cos (\gamma)=\frac{v}{v^{\prime}} \\
\frac{z}{\tan (\gamma)}=x^{\prime} \cos (\gamma) \\
z=x^{\prime} \sin (\gamma)
\end{gathered}
$$

From SI figure $\mathrm{S} 2(\mathrm{~b})$, it is clear that if the beam divergence angle $\theta$ is small enough $\left(\approx 3^{\circ}\right)$ and $\mathrm{z}$ is around the beam waist, the relationship between the beam width in the $\mathrm{x}$ direction $(2 \mathrm{w} x)$ can be related to the beam width in the $\mathrm{x}^{\prime}$ direction through the simple trigonometric formulation:

$$
w_{x}=w_{x} \cos (\gamma)
$$

Since at small enough $\theta, \zeta \approx \delta_{s} \approx \delta_{l}$

And hence from the figure $\mathrm{S} 2(\mathrm{~b}), w_{x^{\prime} \delta s} \approx w_{x^{\prime} \delta l} \approx 0$ 
And $w_{x^{\prime} \zeta s} \approx w_{x^{\prime} \zeta l} \approx w_{x^{\prime}} \approx \frac{w_{x}}{\cos (\gamma)}$

To calculate the new dwell time:

$$
D_{t}\left(x^{\prime}\right)=\frac{2 w_{x^{\prime}}(z)}{v^{\prime}} \approx \frac{2 w_{x}(z) / \cos (\gamma)}{v / \cos (\gamma)}=\frac{2 w_{x}(z)}{v}=D(z)
$$

which is the same as in the untilted lasing case.

- To get the average flux $\mathrm{I}_{\mathrm{av}}$ with spot size on untilted surface:

$$
I_{a v}(z)=\frac{\int_{-w_{x}(z)}^{w_{x}(z)} \int_{w_{y(z)}}^{w_{y}(z)} I(x, y, z) d y d x}{A_{f}(z)}=\frac{I_{o}}{\pi w_{x}(z) w_{y}(z)} \int_{-w_{x}(z)}^{w_{x}(z)} \int_{-w_{y}(z)}^{w_{y}(z)} e^{-2\left(\frac{x}{w_{x}(z)}\right)^{2}} e^{-2\left(\frac{y}{w_{y}(z)}\right)^{2}} d x d y
$$

- To get the average flux Iav,t with spot size on tilted surface:

$$
I_{a v, t}(z)=\frac{\int_{A_{t}(z)} I^{\prime}\left(x^{\prime}, y, z\right) d x^{\prime} d y}{A_{t}(z)} \approx \frac{\int_{A_{t}(z)} I^{\prime}\left(x^{\prime}, y, z\right) d x^{\prime} d y}{\pi w_{x^{\prime}}(z) w_{y}(z)}
$$

The integration above was evaluated numerically by creating the function $I^{\prime}\left(x^{\prime}, y, z\right)$ using the approach shown in Figure S2(a) and using the dimensions of the tilted spots as integration limits at the angles $\gamma$ used in this study. The results have shown that the estimated averaged fluence at $\mathrm{z}$ :

$$
I_{a v}(z) \approx I_{a v, t}(z)
$$


This can be explained with the fact that since the tilt angle range doesn't drastically change the beam intensity profile, the beam broadens but so does the beam spot so averaging over the area doesn't affect the averaged intensity over the spot.

To calculate the average fluence:

$$
F(z)=D_{t}(z) I_{a v, t}(z)=D(z) I_{a v}(z)
$$

Hence, the average fluence for the untilted surface is equal to the average fluence of the tilted surface for our range of $\theta$ and $\gamma$. 

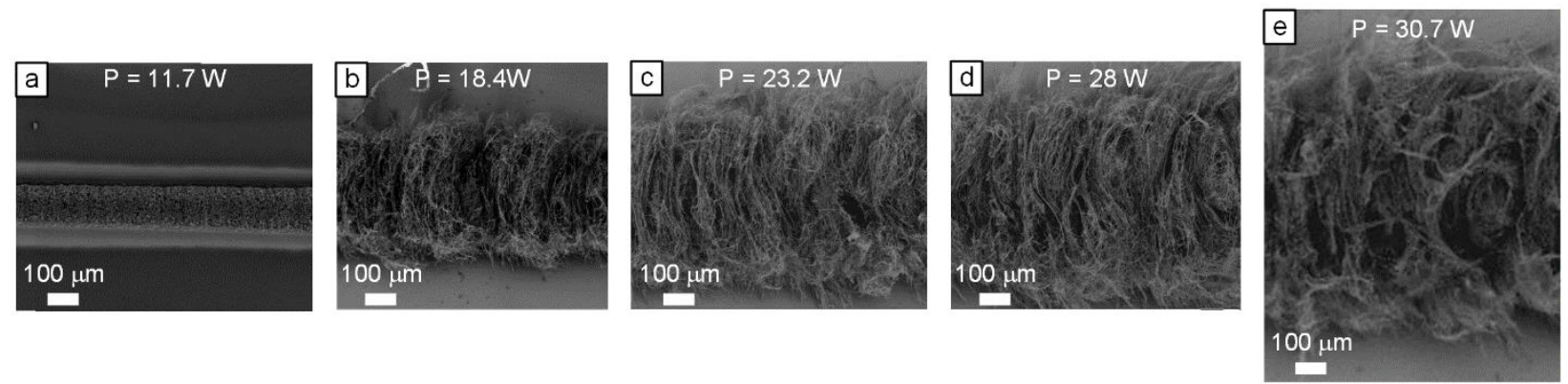

Figure S3. SEM images illustrating the effect of power on line width and woolly fiber volume at powers (a) $\mathrm{P}=11.7 \mathrm{~W}$, (b) $\mathrm{P}=18.4 \mathrm{~W}$, (c) $\mathrm{P}=23.2 \mathrm{~W}$, (d) $\mathrm{P}=28 \mathrm{~W}$, (e) $\mathrm{P}=30.2 \mathrm{~W}$. 

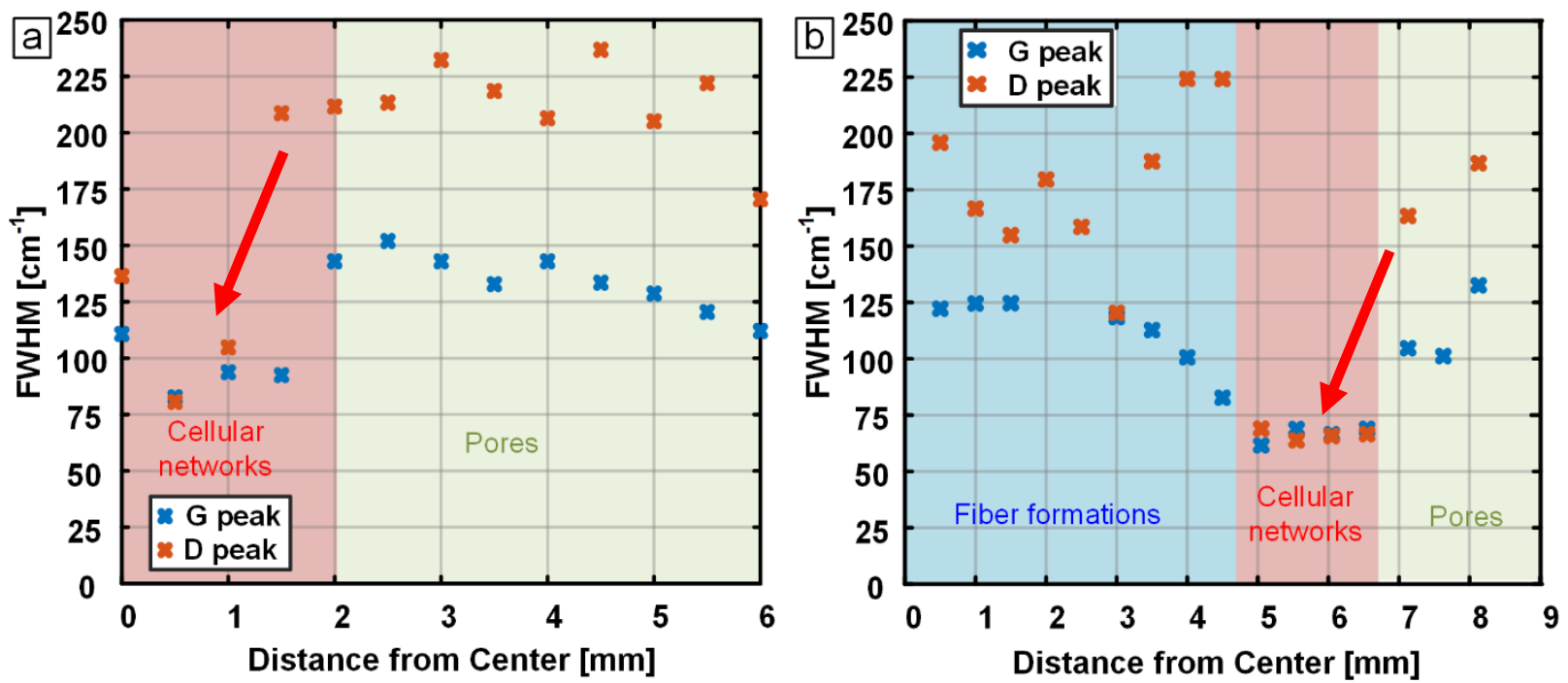

Figure S4. Plot showing the change in FWHM of the G and D peak with changing morphology along a LINC line generated by tilted lasing at $\gamma=45^{\circ}$ at (a) power $\mathrm{P}=11.7 \mathrm{~W}$ and speed $\mathrm{v}=500$ $\mathrm{mm} / \mathrm{s}$ and (b) power $\mathrm{P}=28 \mathrm{~W}$ and speed $\mathrm{v}=500 \mathrm{~mm} / \mathrm{s}$. 


\section{Simulation of surface temperature during lasing at untilted and tilted conditions}

To estimate the change of surface temperature with time and verifying the conclusions of the previous section, we develop a finite element method (FEM) model of the problem. We simulate the laser heating with Gaussian surface flux on the upper surface of the material. The parameters of the Gaussian laser beam were estimated experimentally using laser power measurements and the knife edge method. Polymer lasing is a complex process with laser material interactions, phase and chemical transitions happening at different time scale. The current model is highly simplified, however it should provide some useful insight into the process. Two cases were simulated, a untilted case at different defocus conditions and a tilted case with a tilt angle of 45 degrees as illustrated in Figure S3 $(a, b)$ The boundary conditions utilized are shown in Figure S3 $(a, b)$. The simulations were conduced using the FEM program ANSYS APDL. In the simulation, we exploit the half symmetry of the problem by only modeling half the geometry.

Convection boundary conditions $\left(\mathrm{Q}_{\text {conv }}\right)$ were applied on the upper and lower surface with a heat transfer coefficient of $h_{1}=17.9 \mathrm{~W} / \mathrm{m}^{2} / \mathrm{K}$ and $\mathrm{h}_{2}=9.0 \mathrm{~W} / \mathrm{m}^{2} / \mathrm{K}$ for the upper and lower surface respectively. $^{1}$

The materials properties were assumed to be temperature dependent to take into account some of the chemical changes happening in the process. Temperature dependent material estimated from studies on the thermal properties of polyimide and graphitic material. The temperature dependent thermal properties are illustrated in Figure S3 (c).

The temperature dependent thermal conductivity $\mathrm{K}$ is estimated to $\mathrm{be}^{2}$ : 
$\mathrm{K}=\left\{\begin{array}{c}0.213+3.416 * 10^{-5} T[W /(m K)], 200 K<T<729 K \\ -1.314+2.13 * 10^{-3} T[W /(m K)], 729 K<T<1500 K \\ 1.88[W /(m K)], T>1500 K\end{array}\right.$

The heat capacity $\left(\mathrm{C}_{\mathrm{p}}\right)$ is modeled piecewise with the thermal properties of polyimide as well as amorphous carbon as ${ }^{3-5}$ :

$$
\mathrm{C}_{p}=\left\{\begin{array}{c}
1000\left(0.96+1.39\left(\frac{T-300}{400}\right)-0.42\left(\frac{T-300}{400}\right)^{2}\right)[\mathrm{J} /(K g K)], 200 K<T<915 K \\
2080[\mathrm{~J} /(\mathrm{Kg} K)], 915 K<T<1800 \mathrm{~K} \\
-0.000143 T^{2}+0.734 T+1220[\mathrm{~J} /(\mathrm{Kg} \mathrm{K})], 1800 \mathrm{~K}<T
\end{array}\right.
$$

Radiation boundary conditions $\left(\mathrm{Q}_{\mathrm{rad}}\right)$ were applied on the upper surface and an emmisivity of 0.8 was assumed on the upper surface. The material thickness used is $50 \mu \mathrm{m}$ the width was $500 \mu \mathrm{m}$. The length of the tilted case is $11 \mathrm{~mm}$ Hexahedron elements were utilized to mesh the geometry. A mesh number of 12000 is used for the untilted case and a mesh number of 15000 for the tilted case. A sample mesh is shown in Figure S3 (d). 


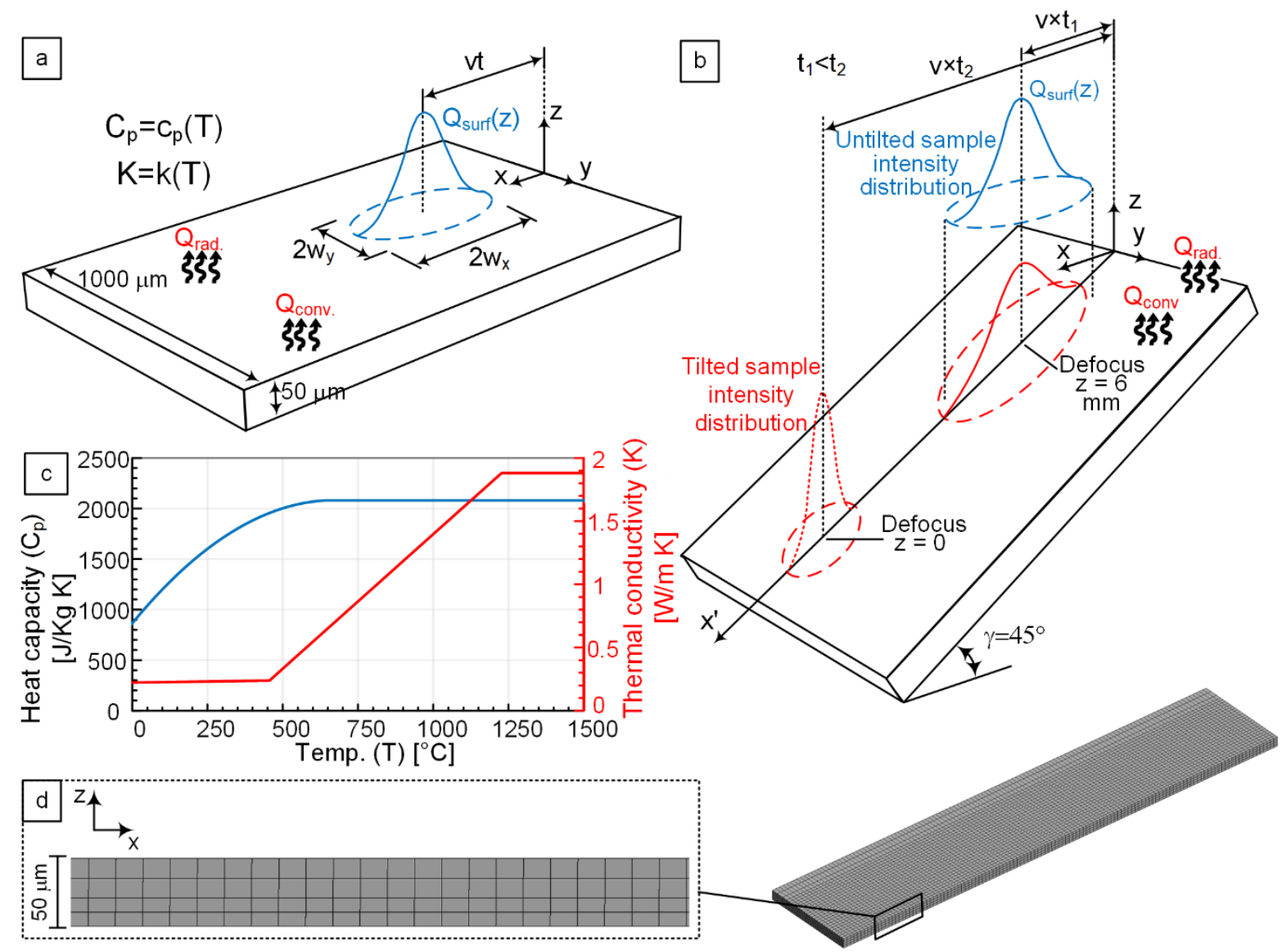

Figure S5. (a) Illustration of the untilted case and the boundary conditions including the Gaussian flux distribution (b) Illustration of the tilted case and the boundary conditions including the Gaussian flux distribution (c) Temperature dependent thermal properties of polyimide carbonizing (d) Mesh used to model untilted condition.

Surface flux was applied using the following equations describing an elliptical Gaussian beam:

$$
I(x, y, z)=\frac{2 P}{\pi w_{x}(z) w_{y}(z)} e^{-2\left(\left(\frac{x-v t}{w_{x}(z)}\right)^{2}+\left(\frac{y}{w_{y(z)}}\right)^{2}\right)}\left[\frac{W}{m^{2}}\right]
$$




$$
\begin{aligned}
& w_{x}(z)=w_{o x} \sqrt{1+\left(\frac{M_{x}^{2} \lambda z}{\pi w_{o x}^{2}}\right)^{2}} \\
& w_{y}(z)=w_{o y} \sqrt{1+\left(\frac{M_{y}^{2} \lambda z}{\pi w_{o y}^{2}}\right)^{2}}
\end{aligned}
$$

Where $\mathrm{P}$ is the laser power. $\mathrm{W}_{\mathrm{x}}$ and $\mathrm{w}_{\mathrm{y}}$ are the beam dimensions. $\mathrm{M}_{\mathrm{x}}{ }^{2}$ and $\mathrm{My}_{\mathrm{y}}{ }^{2}$ are the beam quality factor for the $\mathrm{x}$ and $\mathrm{y}$ direction respectively, wox and $\mathrm{w}_{\mathrm{oy}}$ are the beam waist dimensions. $\mathrm{v}$ is the velocity of the laser beam which scans along the symmetry plane.

For post-processing, we probe the surface temperature of a point along the symmetry plane with time. For the untilted case, we probe points far downstream of the laser path after insuring that the temporal profile doesn't change with the probed point. This is done at different levels of defocus (z). For the tiled case, we also probe the surface temperature of points along the symmetry plane with time at equivalent levels of defocus $(\mathrm{z})$. The results at different level of defocus $\mathrm{z}$ are illustrated in Figure S4 (a,b). The temperature contours at the surface are shown in Figure S4 (c,d). 

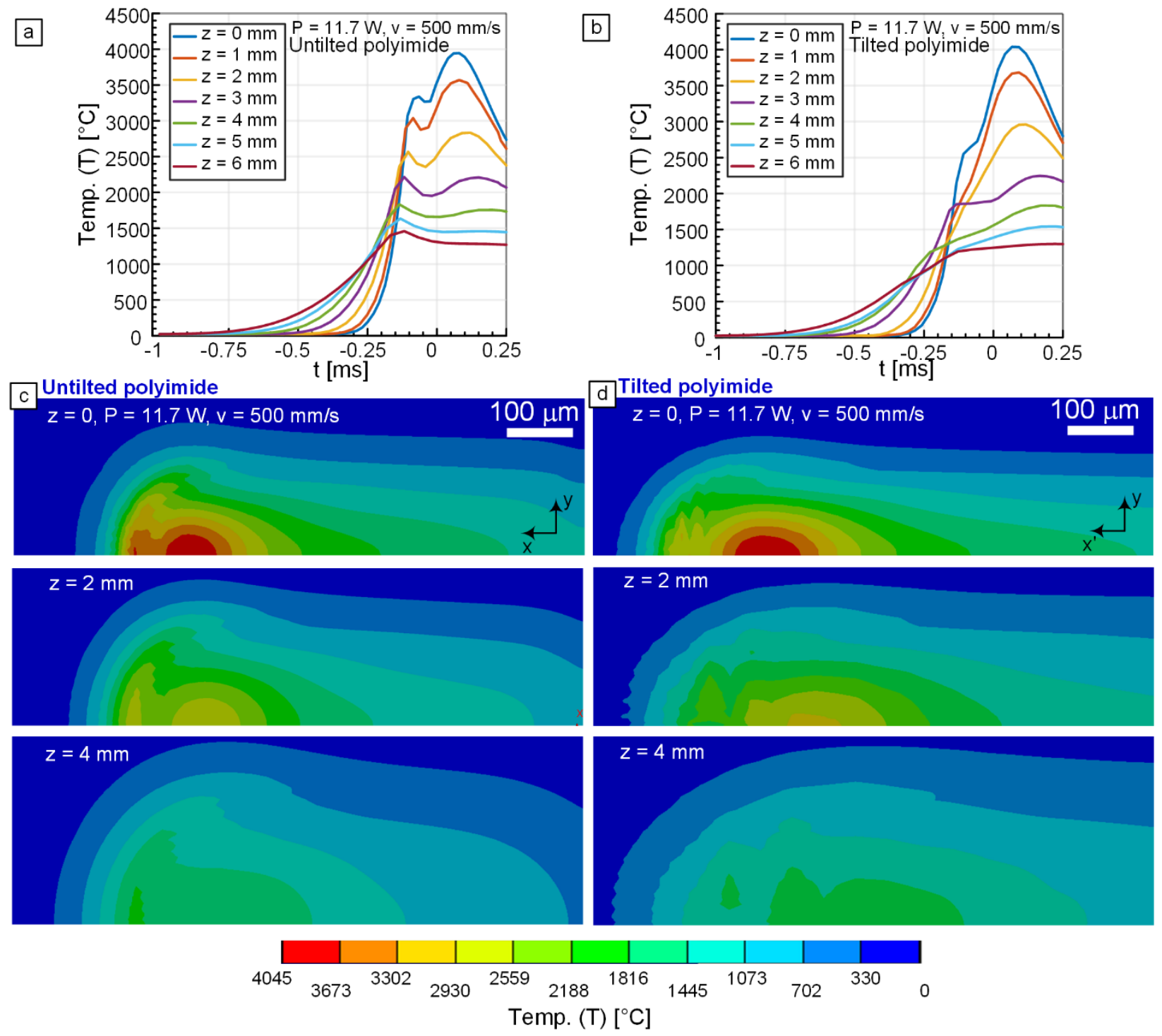

Figure S6. (a) Surface temperature probe at the untilted condition at different levels of defocus for $\mathrm{P}=11.7 \mathrm{~W}$ and $\mathrm{v}=500 \mathrm{~mm} / \mathrm{s}$. Time $\mathrm{t}=0$ is when the center of the beam coincides with the probed point. (b) Surface temperature probe at the tilted condition at different levels of defocus for $\mathrm{P}=$ 11.7 $\mathrm{W}$ and $\mathrm{v}=500 \mathrm{~mm} / \mathrm{s}$. Time $\mathrm{t}=0$ is when the center of the beam coincides with the probed point. (c) Temperature contours at the untilted condition at different levels of defocus at a $\mathrm{P}=11.7$ 
$\mathrm{W}$ and $\mathrm{v}=500 \mathrm{~mm} / \mathrm{s}(\mathrm{d})$ Temperature contours at the tilted condition at different levels of defocus at a $\mathrm{P}=11.7 \mathrm{~W}$ and $\mathrm{v}=500 \mathrm{~mm} / \mathrm{s}$.

When probing the surface temperature at $\mathrm{z}=0$ for both the untilted and tilted case, it is noted that the maximum temperature is around $3945{ }^{\circ} \mathrm{C}$ and $4040^{\circ} \mathrm{C}$ respectively and the maximum temperatre rate $(\mathrm{dT} / \mathrm{dt})$ is $3.153 \times 10^{7}{ }^{\circ} \mathrm{C} / \mathrm{s}$ and $2.258 \times 10^{7}{ }^{\circ} \mathrm{C} / \mathrm{s}$ respectively as shown in Figure $\mathrm{S} 5$ (Left). The temperature of the tilted case is very close to the untilted case within $2.35 \%$. The tilted rate is within $28.3 \%$ of the untilted rate. The temperature distribution is slightly different between the untilted and the tilted, with the tilted case having a more uniform temperature distribution. This can be explained by how the applied fleunce is experienced by the probed points. For the tilted case, the fluence is ramped along the lasing path, while with the untilted case the fluence is constant across the path. This along with the temperature dependence of the material and material thermal diffusivity can lead to the difference between the two cases in temperature, rate and distribution. Overall, when monitoring the maximum temperature at different levels of defocus (shown in Figure S5, right), it is noted that the temperature are very close until around a level of defocus (z) of around $4 \mathrm{~mm}$ and then they start to slightly diverge. 

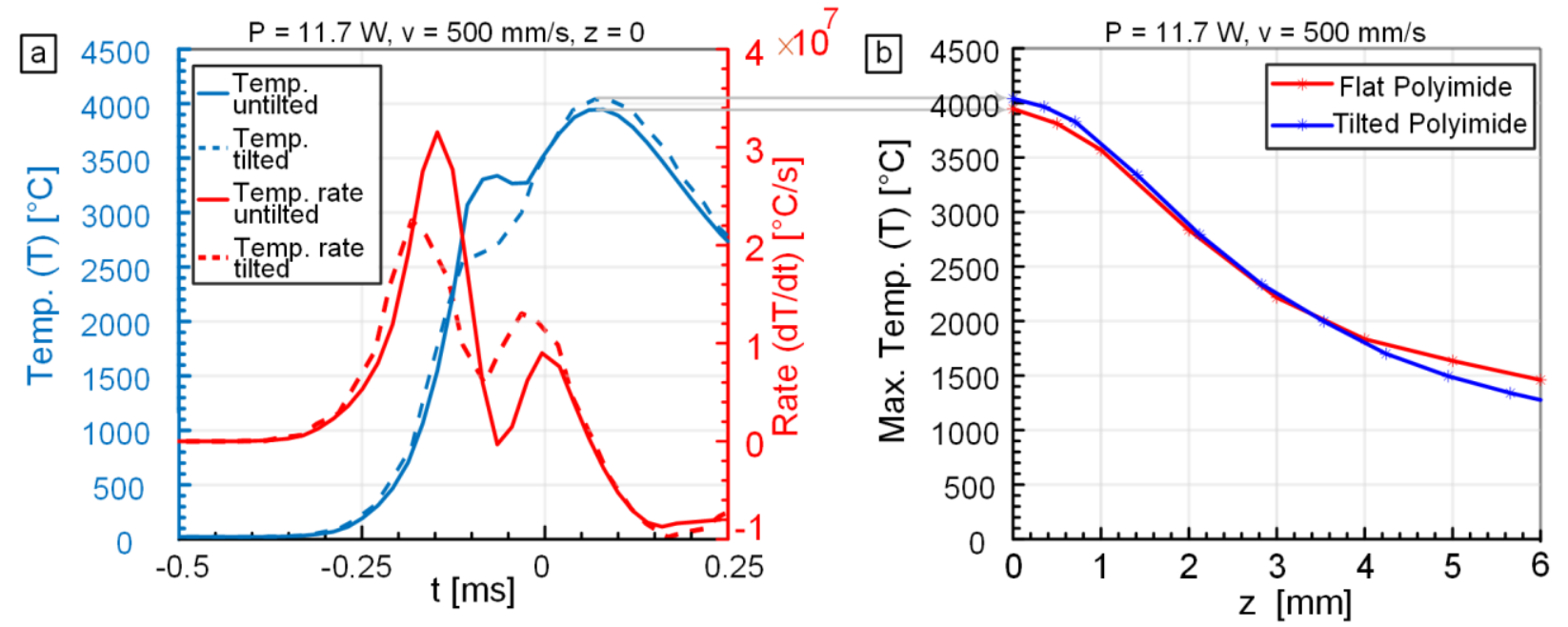

Figure S7. (a) Surface temperature of polyimide for the untilted and the tilted case in addition to the temperature rate. Time $t=0$ represents when the center of the beam coincides with the probed point. (b) Plot of the maximum temperature at the different levels of defocus.

The simulation results do support the conclusions of the previous section that the average fluence is not influenced by the tilt for similar defocus levels. Here, the maximum temperature experienced along the lased path have close values for both the untilted at tilted case at similar defocus levels.

The values of the temperature reported here are difficult to experimentally verify but within range other reported simulation temperatures. ${ }^{1,3}$ 


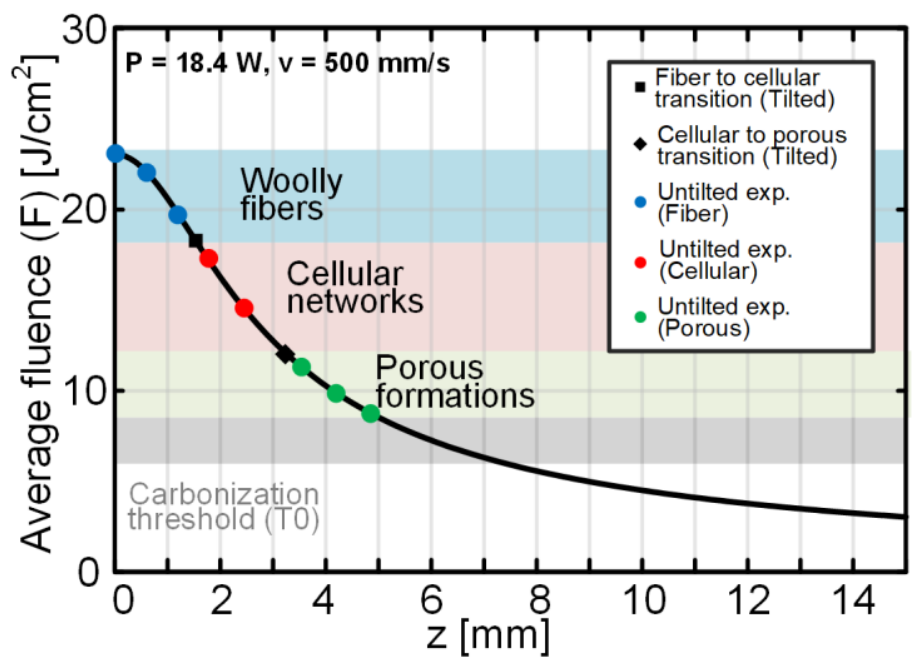

Figure S8. The average fluence experimenced by the substrate at $\mathrm{P}=18.4 \mathrm{~W}$ and $\mathrm{v}=500 \mathrm{~mm} / \mathrm{s}$ at different level of defocus $(\mathrm{z})$, the transitions from wolly fibers to cellular networks and from cellular to porous structures (calculated form tilted lasing experiments) are illustrated. Additionally, the morphologies experienced at different $\mathrm{z}$ values from the untilted lasing experiments shown in Figure 5 are illustrated as well. 

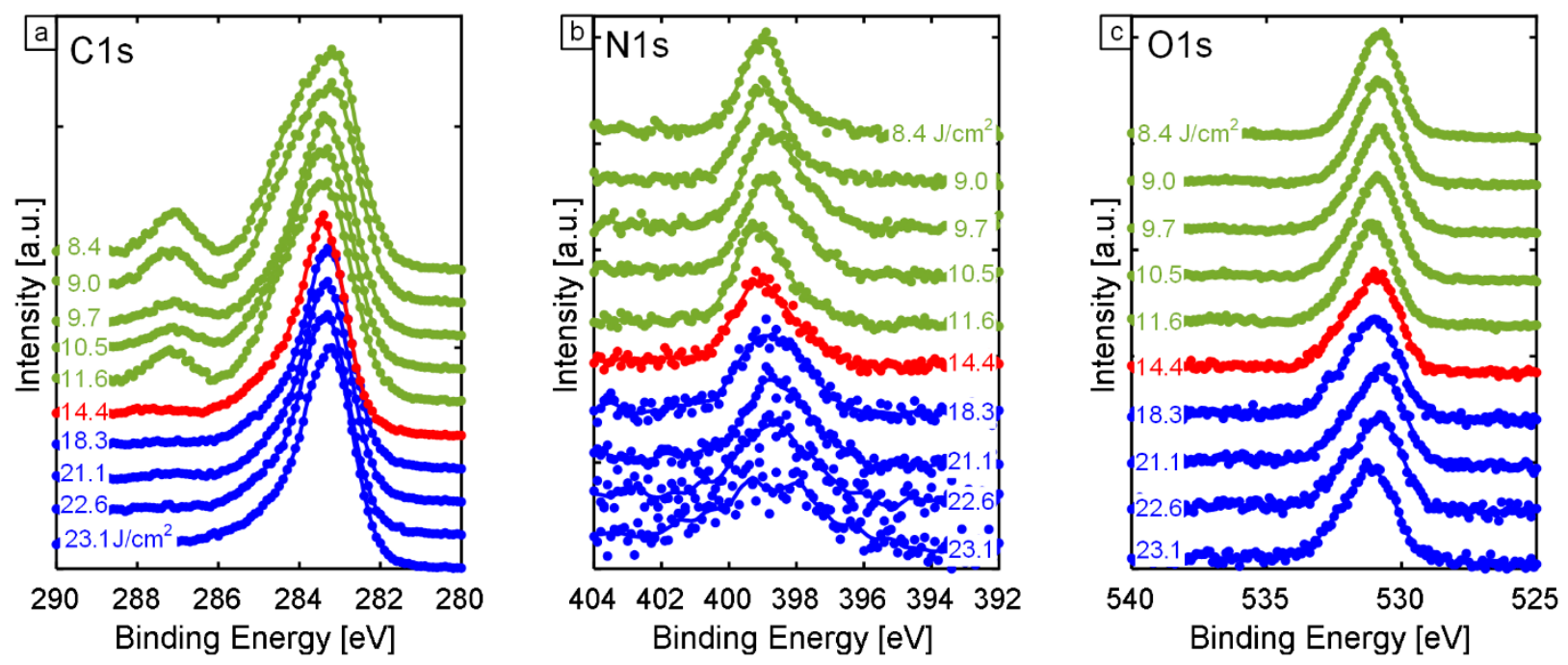

Figure S9. XPS results for several point across an individual LINC line generated by tilted lasing at $\gamma=45^{\circ}$ at power $\mathrm{P}=18.4 \mathrm{~W}$ and speed $\mathrm{v}=500 \mathrm{~mm} / \mathrm{s}$. (a) C1s core scans, (b) N1s core scans, and (c) O1s core scans. Fluence values are indicated on each spectrum in (a). Note the disappearance of the broad band between 286 and $288 \mathrm{eV}$ in (a), which indicates that rapid loss of heteroatoms after T1. All green lines represent isotropic porous morphology, while the red spectrum represents anisotropic networked morphology, and the blue spectra represent aligned nanofibers. 
Table S1: Table of atomic \% of carbon, nitrogen and oxygen as evaluated from XPS survey scans of points along center of lines lased on tilted polyimide sample $\left(\gamma=45^{\circ}\right)$ at $\mathrm{P}=11.7 \mathrm{~W}$. Fluence values are estimated at each point using the beam model.

\begin{tabular}{|c|c|c|c|c|}
\hline$X^{\prime}$ location [um] & Fluence $(\mathrm{F})\left[\mathrm{J} / \mathrm{cm}^{2}\right.$ ] & Carbon [at. \%] & Nitrogen [at. \%] & Oxygen [at. \%] \\
\hline 0.00 & 14.71 & 81.34 & 6.94 & 11.72 \\
\hline 552.60 & 14.30 & 79.34 & 6.68 & 13.99 \\
\hline 838.60 & 13.84 & 74.30 & 7.39 & 18.31 \\
\hline 1424.30 & 12.66 & 71.75 & 9.43 & 18.82 \\
\hline 1835.40 & 11.73 & 71.97 & 8.48 & 19.54 \\
\hline 2336.30 & 10.62 & 72.53 & 8.82 & 18.65 \\
\hline 2714.80 & 9.82 & 72.18 & 7.87 & 19.95 \\
\hline 3297.80 & 8.72 & 68.76 & 5.54 & 25.70 \\
\hline 3624.90 & 8.17 & 66.30 & 6.49 & 27.21 \\
\hline 4156.80 & 7.39 & 64.98 & 5.82 & 29.20 \\
\hline
\end{tabular}

Table S2: Table of atomic \% of carbon, nitrogen and oxygen as evaluated from XPS survey scans of points along center of lines lased on tilted polyimide sample $\left(\gamma=45^{\circ}\right)$ at $\mathrm{P}=18.4 \mathrm{~W}$. Fluence values are estimated at each point using the beam model.

\begin{tabular}{|c|c|c|c|c|}
\hline$X^{\prime}$ location [um] & Fluence $(\mathrm{F})\left[\mathrm{J} / \mathrm{cm}^{2}\right]$ & Carbon [at. \%] & Nitrogen [at. \%] & Oxygen [at. \%] \\
\hline 0.00 & 23.11 & 89.91 & 3.07 & 7.01 \\
\hline 482.90 & 22.60 & 89.62 & 3.54 & 6.84 \\
\hline 1043.80 & 21.14 & 87.43 & 4.16 & 8.41 \\
\hline 1861.90 & 18.34 & 85.80 & 4.46 & 9.74 \\
\hline 3038.70 & 14.44 & 83.37 & 5.07 & 11.56 \\
\hline 4141.20 & 11.64 & 70.61 & 7.23 & 22.16 \\
\hline 4727.60 & 10.51 & 72.73 & 7.27 & 20.00 \\
\hline 5226.00 & 9.70 & 69.14 & 6.50 & 24.36 \\
\hline 5722.40 & 9.01 & 61.85 & 6.51 & 31.64 \\
\hline 6272.70 & 8.35 & 61.66 & 6.77 & 31.57 \\
\hline
\end{tabular}

Table S3: Table of atomic \% of carbon, nitrogen and oxygen as evaluated from XPS survey scans of points along center of lines lased on tilted polyimide sample $\left(\gamma=45^{\circ}\right)$ at $\mathrm{P}=23.2 \mathrm{~W}$. Fluence values are estimated at each point using the beam model. 


\begin{tabular}{|c|c|c|c|c|}
\hline$X^{\prime}$ location $[\mathrm{um}]$ & Fluence $(\mathrm{F})\left[\mathrm{J} / \mathrm{cm}^{2}\right]$ & Carbon [at. \%] & Nitrogen [at. \%] & Oxygen [at. \%] \\
\hline 3185.20 & 17.70 & 85.28 & 4.87 & 9.85 \\
\hline 4075.00 & 14.89 & 78.26 & 6.36 & 15.39 \\
\hline 4378.60 & 14.10 & 84.12 & 4.08 & 11.79 \\
\hline 4644.30 & 13.46 & 77.48 & 5.77 & 16.75 \\
\hline 5342.80 & 12.04 & 74.49 & 6.68 & 18.83 \\
\hline 6538.80 & 10.18 & 68.67 & 7.22 & 24.11 \\
\hline 7609.40 & 8.91 & 68.52 & 5.98 & 25.51 \\
\hline 9116.70 & 7.50 & 65.84 & 6.55 & 27.61 \\
\hline 2062.30 & 22.27 & 89.62 & 2.47 & 7.91 \\
\hline
\end{tabular}

Table S4: Table of atomic \% of carbon, nitrogen and oxygen as evaluated from XPS survey scans of points along center of lines lased on tilted polyimide sample $\left(\gamma=45^{\circ}\right)$ at $\mathrm{P}=28 \mathrm{~W}$. Fluence values are estimated at each point using the beam model.

\begin{tabular}{|c|c|c|c|c|}
\hline $\mathrm{X}$ ' location [um] & Fluence $(\mathrm{F})\left[\mathrm{J} / \mathrm{cm}^{2}\right]$ & Carbon [at. \%] & Nitrogen [at. \%] & Oxygen [at. \%] \\
\hline 2600.30 & 24.20 & 89.21 & 4.10 & 6.69 \\
\hline 3563.50 & 19.90 & 86.42 & 2.40 & 11.18 \\
\hline 4134.80 & 17.85 & 86.06 & 3.65 & 10.30 \\
\hline 4617.50 & 16.40 & 82.89 & 4.71 & 12.41 \\
\hline 5227.40 & 14.85 & 73.70 & 8.30 & 17.99 \\
\hline 5956.50 & 13.35 & 70.47 & 8.01 & 21.52 \\
\hline 6793.80 & 11.94 & 67.52 & 6.93 & 25.56 \\
\hline 7534.20 & 10.90 & 68.91 & 7.03 & 24.06 \\
\hline 8741.00 & 9.47 & 64.34 & 8.71 & 26.96 \\
\hline
\end{tabular}



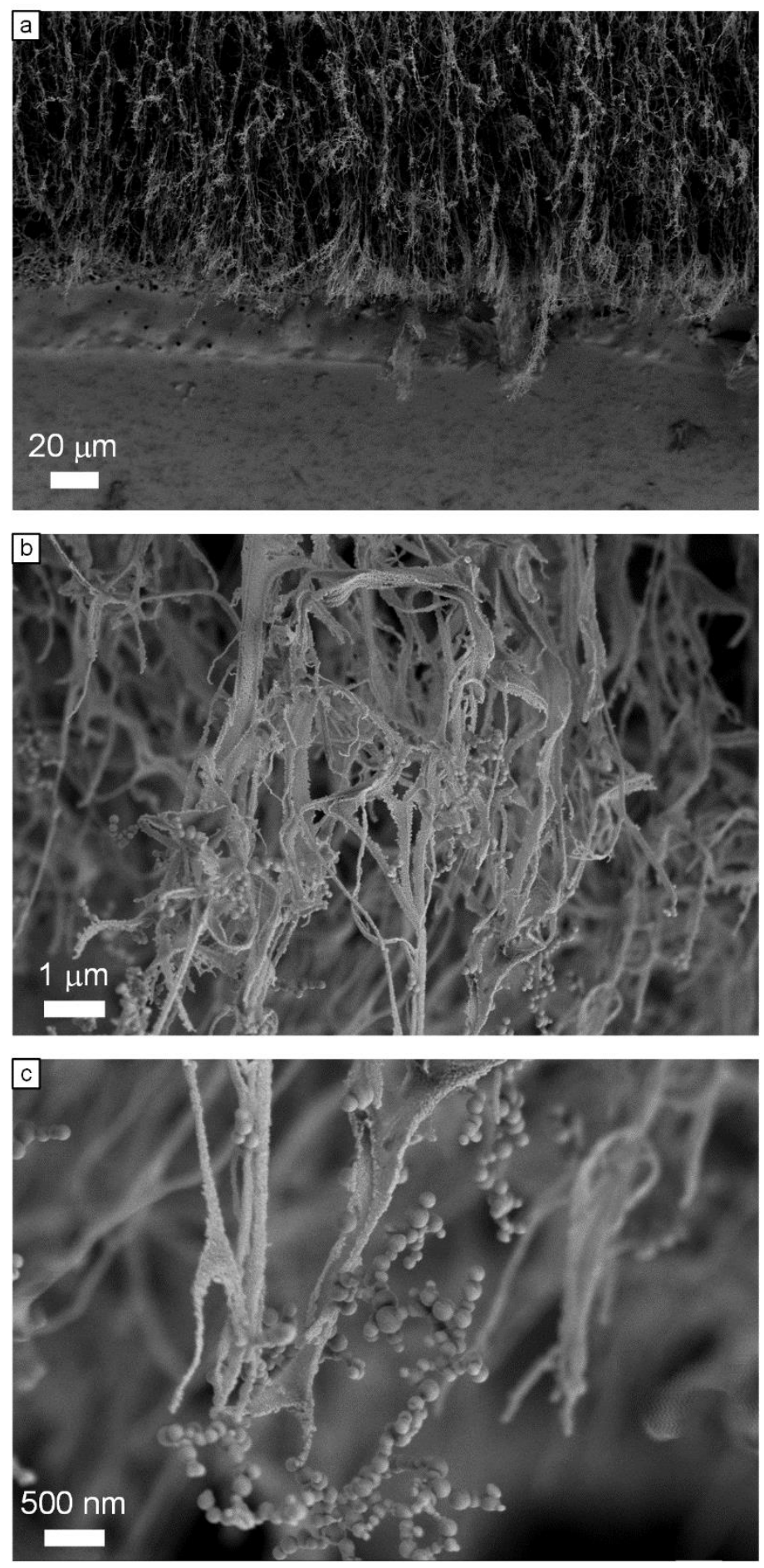

Figure S10. SEM images illustrating the formation of nanospheres at (a) 1000X, (b) 25000X, (c)50000X which suggest melting and liquid beading driven by surface energy minimization. 

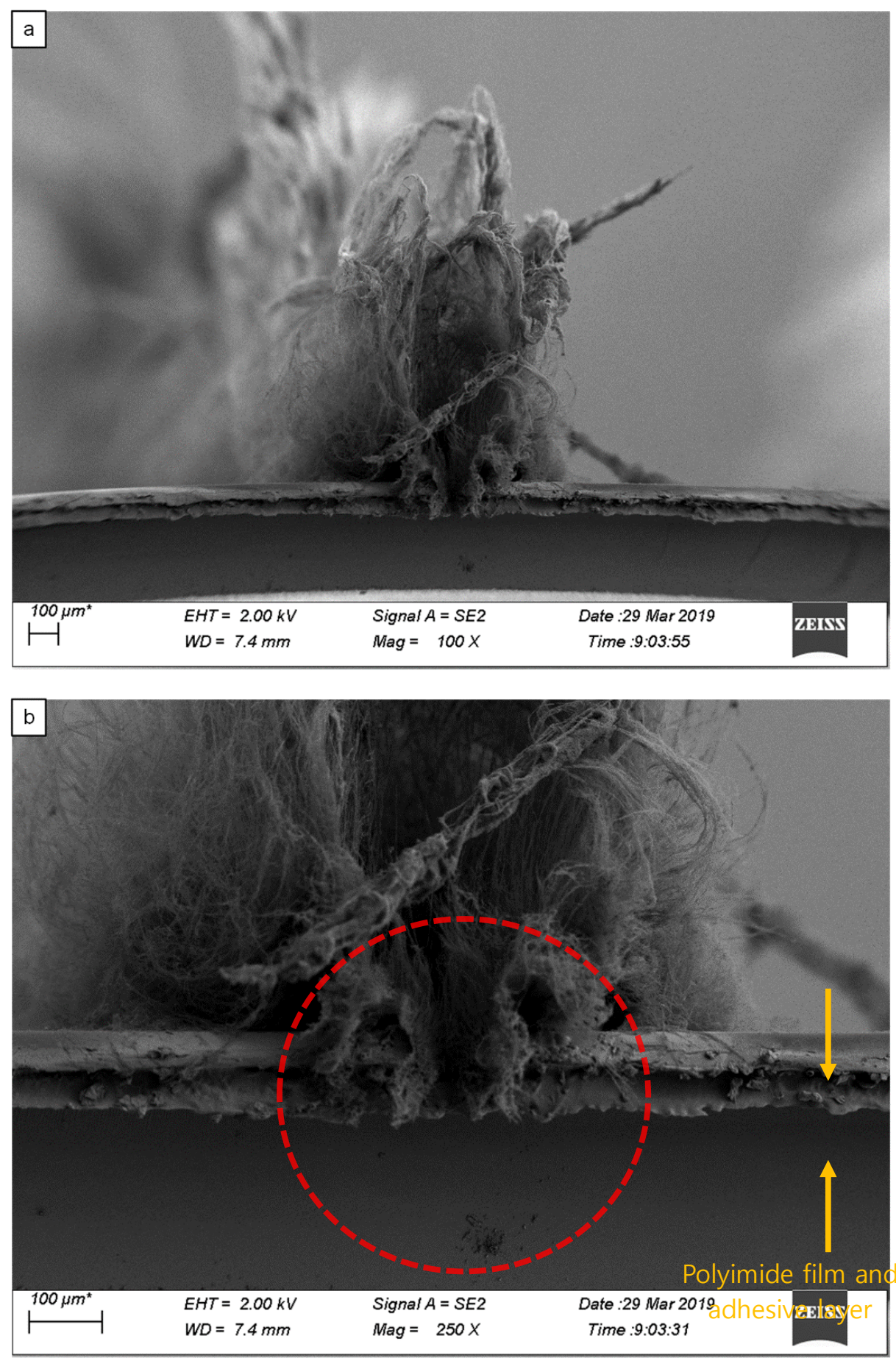

Figure S11. SEM images illustrating the splitting of the polyimide with the formation of the fibrous morphology at magnification (a) $100 \mathrm{X}$ and (b) $250 \mathrm{X}$. Lines lased at $P=30 \mathrm{~W}, v=500 \mathrm{~mm} / \mathrm{s}$, and $z=0 \mathrm{~mm}$. with fluence of $38.2 \mathrm{~J} / \mathrm{cm}^{2}$. 

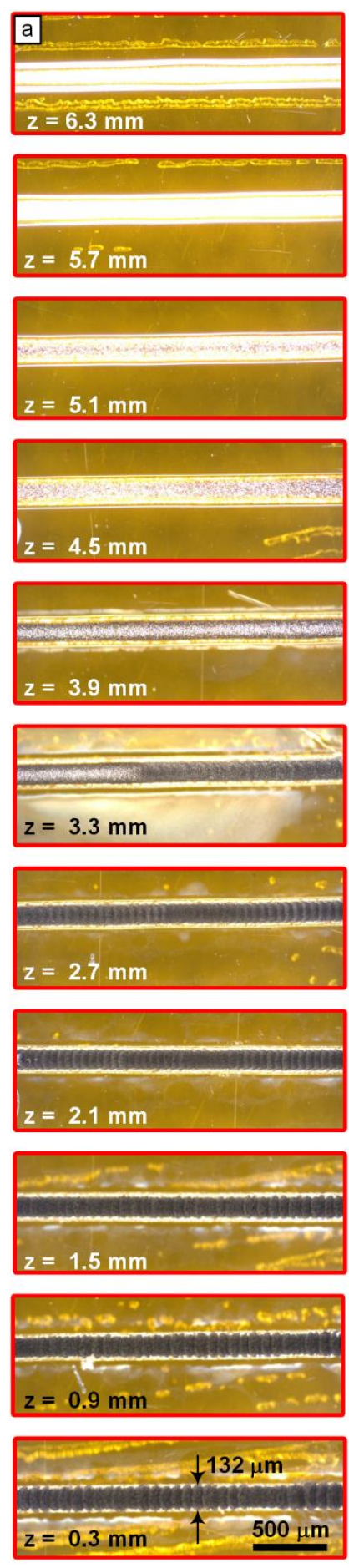

Figure S12. Optical microscope images of the lines used to measure resistance, at (a) $\mathrm{P}=11.7 \mathrm{~W}$ and (b) $\mathrm{P}=28.0 \mathrm{~W}$
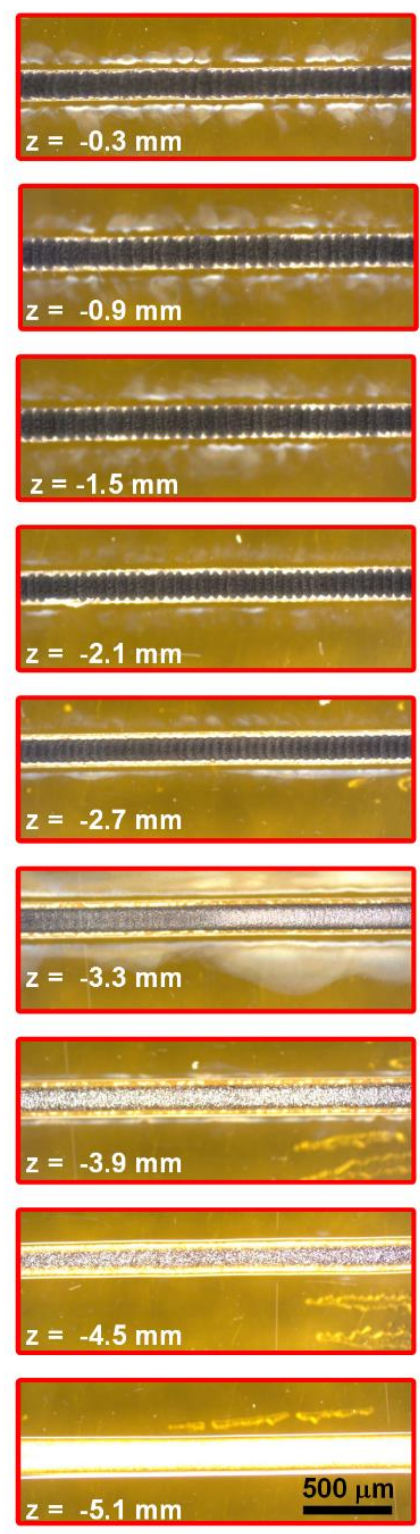
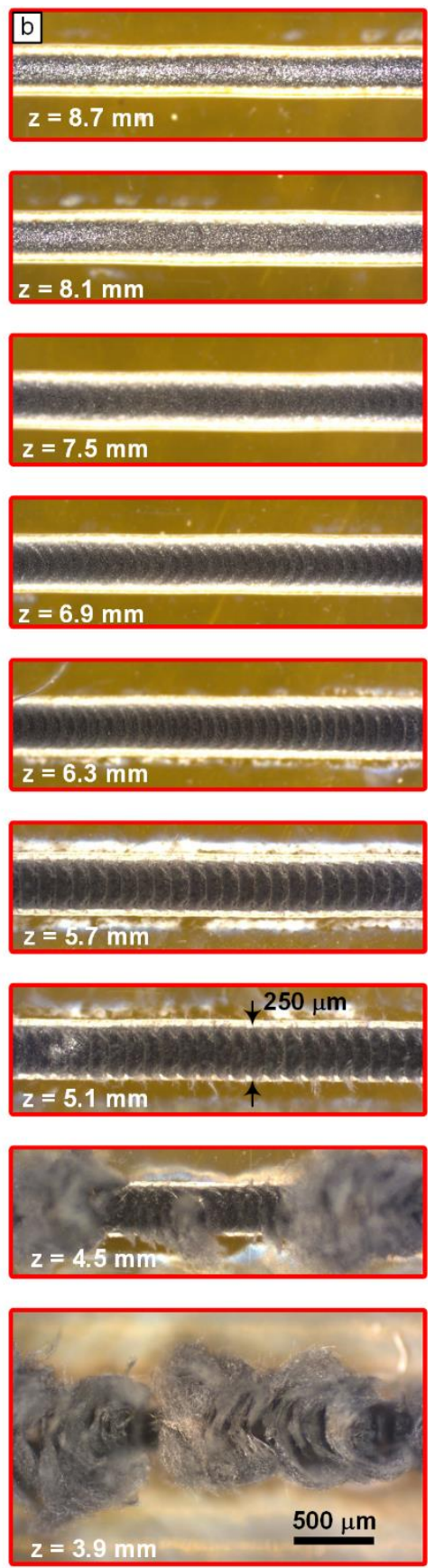

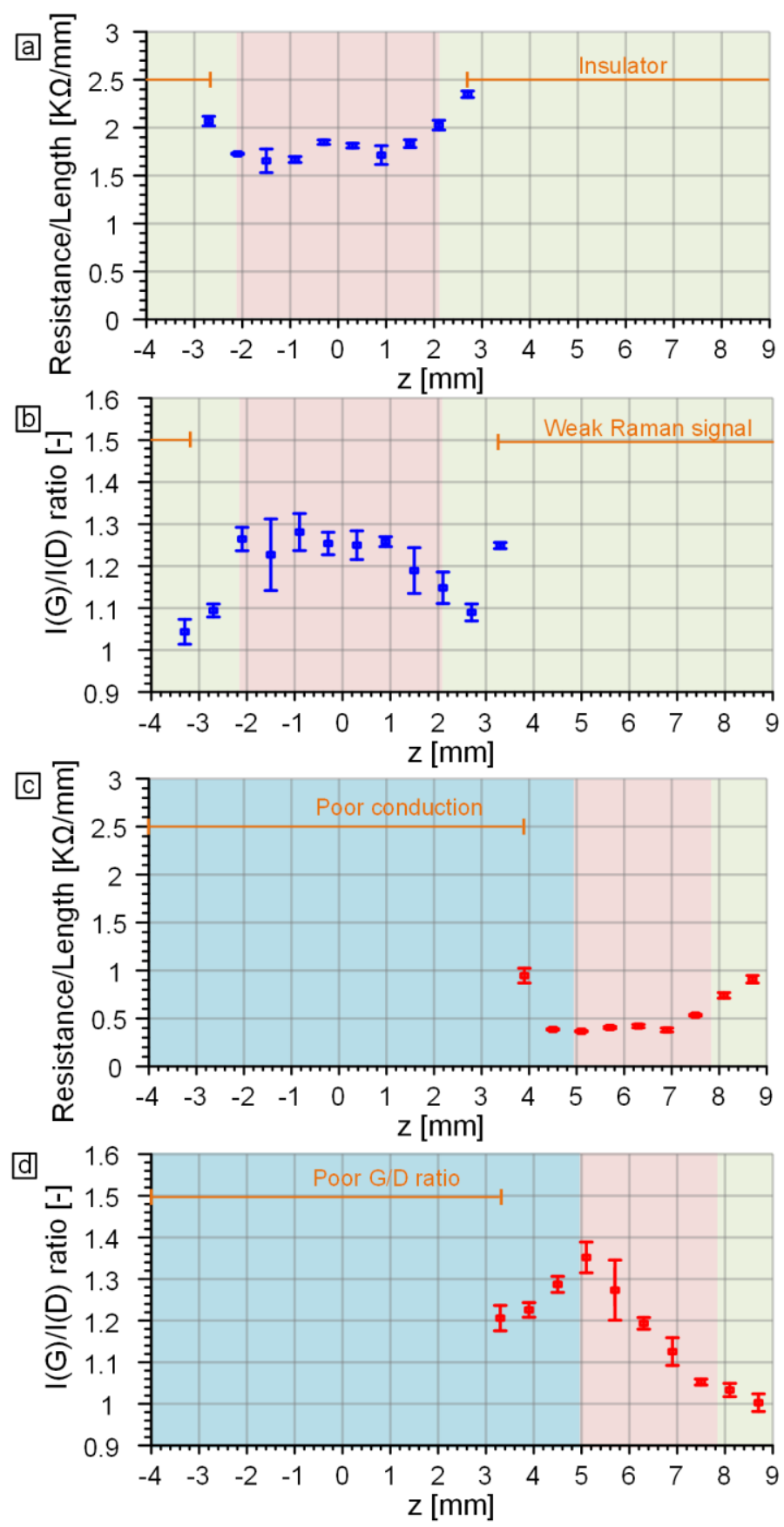

Figure S13. Plot illustrating the change in resistance and $I(G) / I(D)$ in the lased samples showing (a) the resistance per length at $\mathrm{P}=11.7 \mathrm{~W}$, (b) the $\mathrm{I}(\mathrm{G}) / \mathrm{I}(\mathrm{D})$ ratio at $\mathrm{P}=11.7 \mathrm{~W}$, (c) the resistance per length at $\mathrm{P}=28.7 \mathrm{~W},(\mathrm{~d})$ the $\mathrm{I}(\mathrm{G}) / \mathrm{I}(\mathrm{D})$ ratio at $\mathrm{P}=28.7 \mathrm{~W}$. 

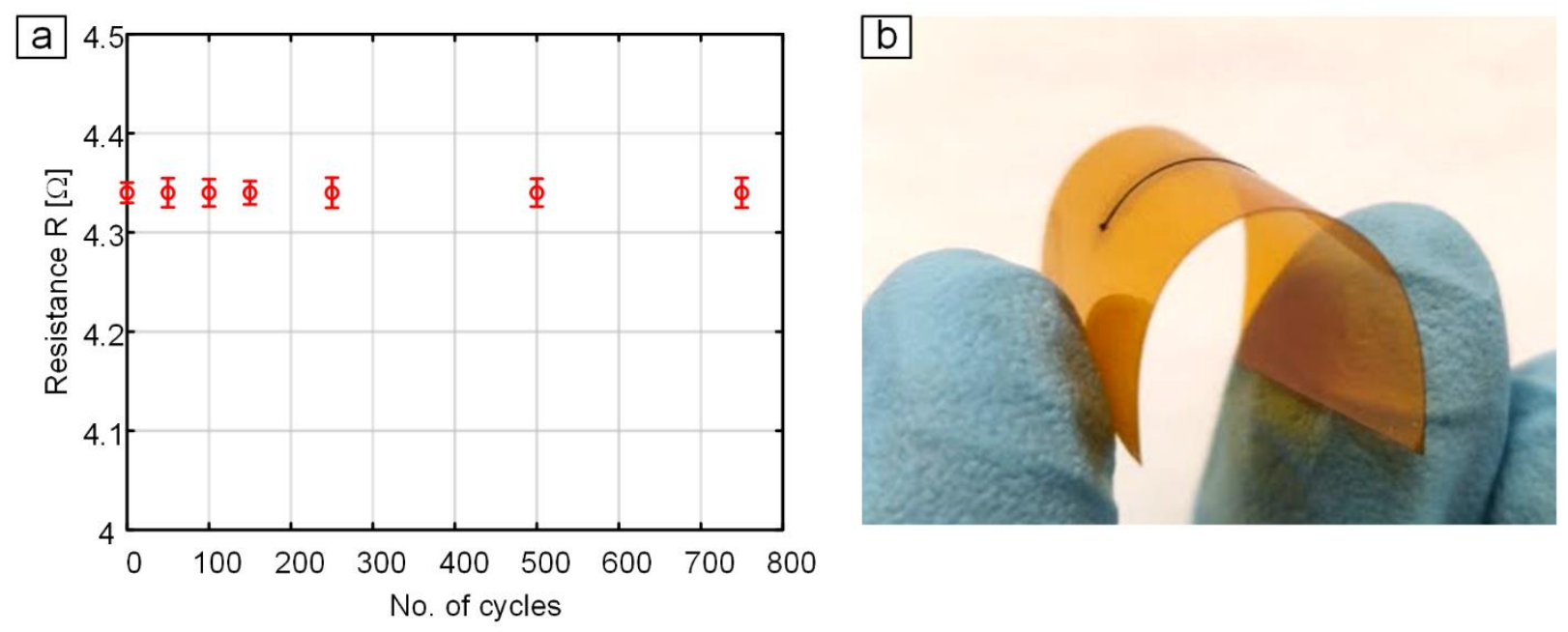

Figure S14. Influence of mechanical bending cycles on LINC electrode resistance, showing stable conductivity up to 750 cycles with no signs of degradation: (a) Measured resistance as a function of the number of bending load cycles for a $10 \mathrm{~mm}$ long LINC line lased at $\mathrm{P}=28 \mathrm{~W}, \mathrm{v}=500 \mathrm{~mm} / \mathrm{s}$, $\mathrm{z}=4 \mathrm{~mm}$. (b) Image of LINC on polyimide film under bending, showing the strong adhesion even after hundreds of bending cycles. 

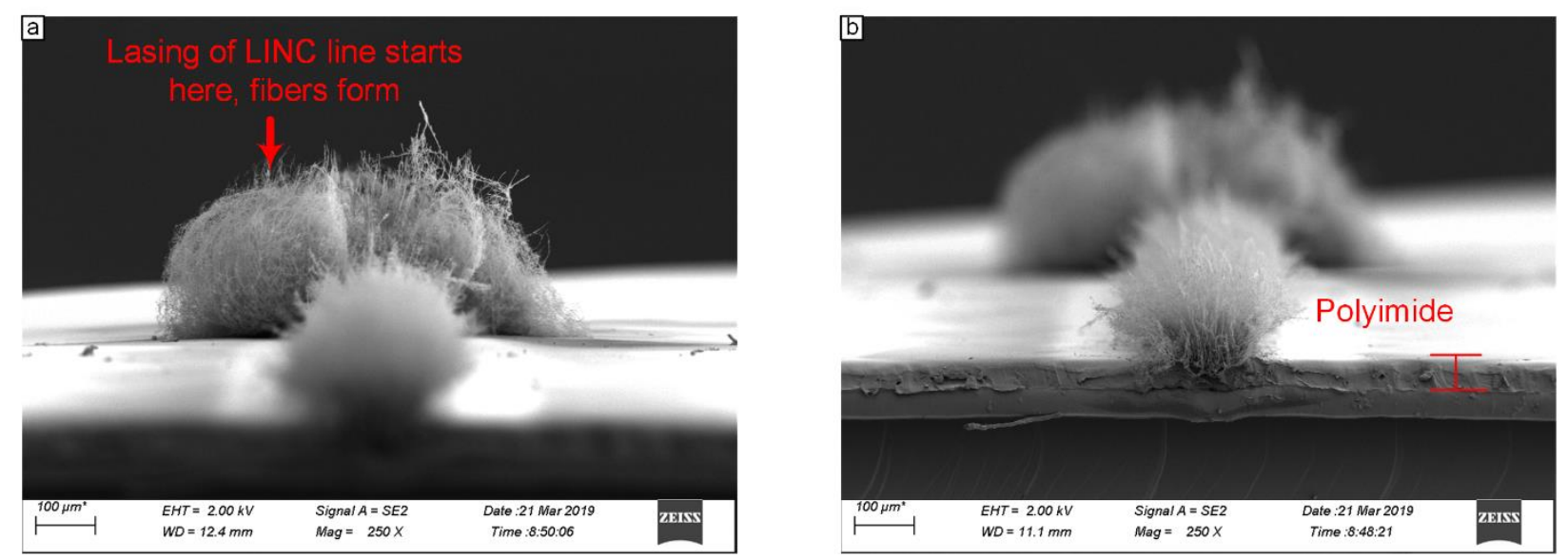

Figure S15. Figure illustrating the influence of laser power transients on lasing of lines where large woolly fibers are formed at (a) the beginning of the line as opposed to (b) the remaining and end of the lased line. It should be noted that this effect is not due to laser head acceleration since it is already in motion when it reached the starting point of lasing. 


\section{Knife Edge method for measuring beam size}

A blade is used to block the assumed Gaussian laser beam at different $\mathrm{z}$ values. The experiment is done with the blade edge parallel to the $\mathrm{y}$ direction of the machine and also the $\mathrm{x}$ direction. The blade is mounted on a precision stage and moved gradually to block the beam. At each blocking distance, the laser is tuned on, where it heats a thermopile system. The power measured at the different blocking distances is used to estimate the value of the $\mathrm{w}_{\mathrm{x}}$ and $\mathrm{w}_{\mathrm{y}}$ (depending on the direction of the blade) at that value of $z$. The data is fit to the curve:

$P_{\text {measured }}=0.5 P_{\text {max }}\left(1+\operatorname{erf}\left(\frac{\sqrt{2} x}{w}\right)\right)$

Repeating this approach multiple times is used to estimate the full shape of the beam, assuming it is elliptical with the principle direction matching the machine $\mathrm{x}, \mathrm{y}$ coordinates. The different width are used to fit this equation:

$$
\begin{aligned}
& w_{x}(z)=w_{o x} \sqrt{1+\left(\frac{M_{x}^{2} \lambda z}{\pi w_{o x}^{2}}\right)^{2}} \\
& w_{y}(z)=w_{o y} \sqrt{1+\left(\frac{M_{y}^{2} \lambda z}{\pi w_{o y}^{2}}\right)^{2}}
\end{aligned}
$$

Where $\mathrm{w}_{\mathrm{ox}}$ and $\mathrm{w}_{\mathrm{oy}}$ are the beam waist spot radii, $\mathrm{M}_{\mathrm{y}}{ }^{2}$ and $\mathrm{M}_{\mathrm{x}}{ }^{2}$ and the beam quality factor.

\section{References:}

(1) Ruan, X.; Wang, R.; Luo, J.; Yao, Y.; Liu, T. Experimental and Modeling Study of CO2 Laser Writing Induced Polyimide Carbonization Process. Mater. Des. 2018, 160, 1168-1177. https://doi.org/10.1016/j.matdes.2018.10.050. 
(2) Venkatachalam, S.; Depriester, M.; Sahraoui, A. H.; Capoen, B.; Ammar, M. R.; Hourlier, D. Thermal Conductivity of Kapton-Derived Carbon. Carbon N. Y. 2017, 114, 134-140. https://doi.org/10.1016/j.carbon.2016.11.072.

(3) Küper, S.; Brannon, J.; Brannon, K. Threshold Behavior in Polyimide Photoablation: Single-Shot Rate Measurements and Surface-Temperature Modeling. Appl. Phys. A Solids Surfaces 1993, 56 (1), 43-50. https://doi.org/10.1007/BF00351902.

(4) Kotel'nikov, G. V.; Sidorovich, A. V. Microcalorimeter Type DSM-2M for Use in the Study of Polymers. Polym. Sci. U.S.S.R. 1983, 25 (12), 3053-3059. https://doi.org/10.1016/00323950(83)90060-6.

(5) Taylor, R. E.; Groot, H. Thermophysical Properties of Poco Graphite; 1978. 